\title{
MAGNETIC AND DOPPLER OSCILLATIONS IN ACTIVE REGIONS
}

\author{
F. L. DEUBNER \\ (Fraunhofer Institut, Freiburg i. Br., Germany)
}

With the Capri magnetograph of the Fraunhofer Institute simultaneous measurements of sightline velocities and longitudinal magnetic fields have been carried out, in order to investigate the dependence of the 300-sec oscillation of sightline velocities on the magnetic-field strengths, and the oscillatory behavior of the latter, if there is any.

The principle of measurement was very similar to that used by Howard and Wilcox (1967) reported at the Prague meeting. The Sun has been scanned repeatedly along a fixed line parallel to the solar axis, with a scanning spot of 3 " diameter. The sensitivity was better than $20 \mathrm{~m} / \mathrm{sec}$ and about 3 gauss (the time constant being $1 \mathrm{sec}$ ).

Since the complete work will be published elsewhere (Deubner, 1967), I shall simply report the results of this investigation without going into the details of the analysis.

The influence of magnetic fields on the lifetime of single bursts of oscillations. Whereas in plage regions as well as in undisturbed regions the most frequent lifetime seems to be about $15 \mathrm{~min}$, a pronounced tendency for longer lifetimes has been found in plage regions.

It seems, that also the mean linear distance of neighbouring oscillating elements is influenced by the presence of magnetic fields. It is about $20 \%$ lower in plage regions than in undisturbed regions, where a value of $8^{\prime \prime}$ has been found. One is reminded of the fact that the granular diameter seems to shrink in the presence of magnetic fields too (Schröter, 1962).

The mean amplitude of 300 -sec oscillations as a function of the magnetic-field strength observed in the oscillating region. From 10 gauss to 100 gauss, the mean amplitudes decrease distinctly from about $600 \mathrm{~m} / \mathrm{sec}$ to $400 \mathrm{~m} / \mathrm{sec}$. It cannot be decided whether the decrease continues towards still higher field strengths.

It seems safe to conclude from our observations, that the period of oscillation is independent of the field strength at least up to several 100 gauss.

It can be seen from velocity records of disturbed regions and simultaneous magneticfield maps that regions, where the oscillations are superimposed by a strong (constant) downward-velocity component, often coincide with stronger magnetic fields. The mean-velocity level for different field strengths has been calculated. There is an unambiguous transition from upward velocities to downward velocities with increasing field strength. This correlation between Doppler shift and magnetic field cannot be

Kiepenheuer (ed.), Structure and Development of Solar Active Regions, 230-232. C I.A.U. 


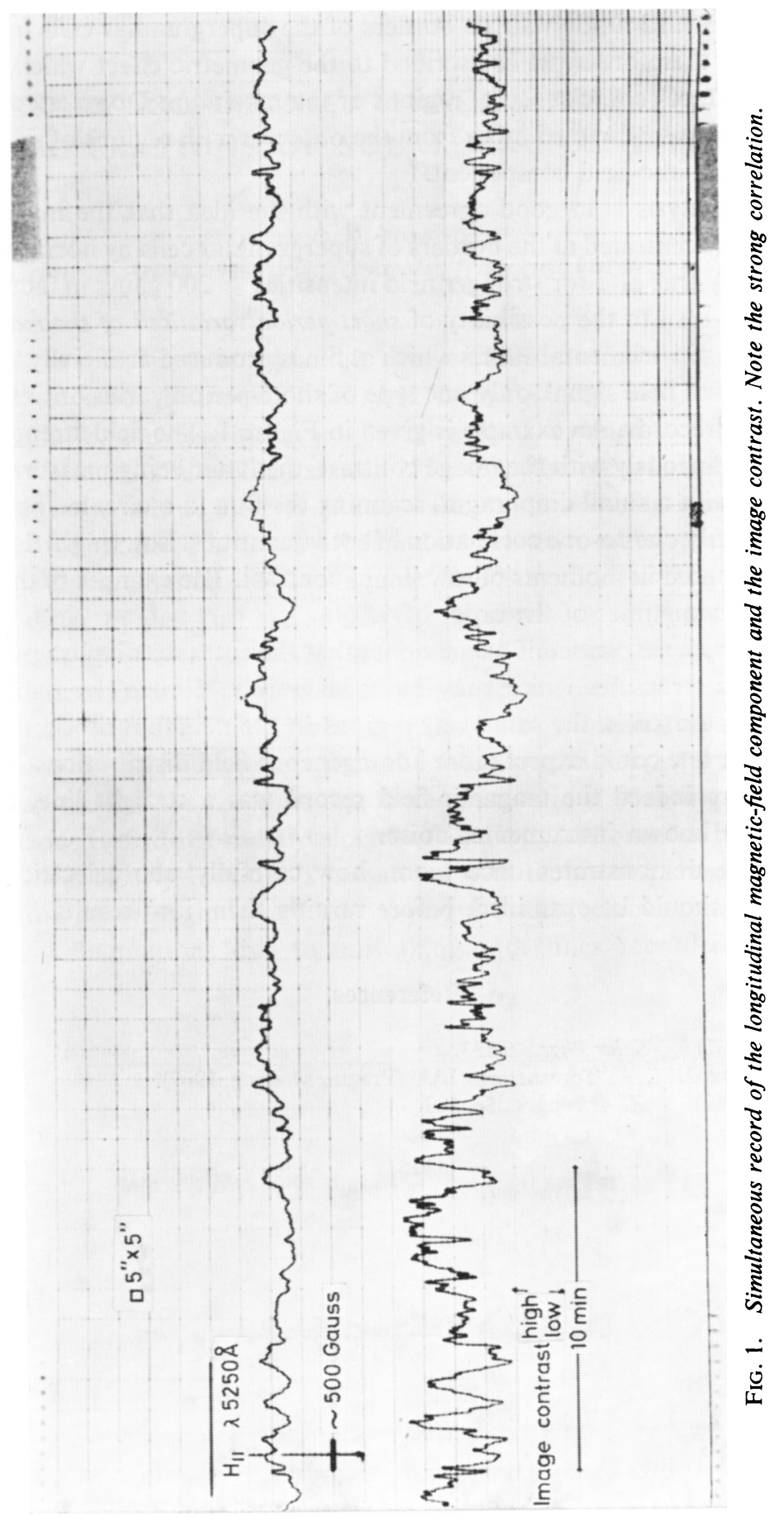


due to the downward motion at the borders of the supergranular cells because of its low velocity. It therefore must be ascribed to the geometric effect which, by oblique projection, produces coincidence of regions of the maximum Doppler component of the horizontal motion directed away from the observer with regions of maxima of the longitudinal magnetic-field component.

This rough analysis is in good agreement with the idea that the magnetic flux is preferentially concentrated at the borders of supergranular cells by horizontal motion. This is especially true also for stronger field intensities $\geqslant 200$ gauss in facular regions.

Finally let us turn to the possibility of short-period variations of the magnetic field. After eliminating instrumental effects which at times produced artificially 'oscillations' of the longitudinal field signal, only one type of short-period variations remained still apparent in the records. An example is given in Figure 1. The field strength has been recorded simultaneously with the image contrast, the latter being measured by means of a photocell with a small diaphragm, scanning the Sun in a circular pattern. There is a striking, nearly one-to-one correlation of both quantities. Low (high) field strengths are always measured in moments of low (high) contrast. The changes of the magneticfield signal are sometimes of the order of $40 \%$ !

This effect may be explained if one assumes that the entrance diaphragm just covers a tiny magnetic structure, which may be considerably 'diluted' by bad seeing. To check this, I measured in the same way regions in the neighbourhood of old stable sunspots, where one could expect more homogeneous field distributions, not affected by seeing. There indeed the magnetic-field record was a straight line, only superimposed by the known instrumental noise.

This example demonstrates once more, how carefully photoelectric records of magnetic fields should be examined, before turning them into results.

\section{References}

Deubner, F.L. (1967) Solar Phys., 2, 133.

Howard, R., Wilcox, J.M. Transactions IAU (Prague Meeting, 1967).

Schröter, E.H. (1962) Z. Astrophys., 56, 183. 